\title{
Ganho Programado Tradicional e Rede de Controladores com Supervisão Fuzzy Aplicados a Sistemas Não-Lineares: um Comparativo
}

\author{
Gustavo Freire de Moura Claude \\ Laboratório de Controle de Sistemas \\ Universidade Federal do Pará \\ Belém, Brasil \\ gustavoclaude@gmail.com
}

\author{
Luis Augusto Mesquita de Castro \\ Laboratório de Controle de Sistemas \\ Universidade Federal do Pará \\ Belém, Brasil \\ luismesquita@ufpa.br
}

\author{
Antonio da Silva Silveira \\ Laboratório de Controle de Sistemas \\ Universidade Federal do Pará \\ Belém, Brasil \\ asilveira@ufpa.br
}

\begin{abstract}
In this work the performance of a traditional gain scheduling and a network of controllers with fuzzy supervision are compared. The analysis is realized on two systems: a nonlinear valve and a Motor-Tachogenerator plant. The gain scheduling is a control strategy in which the parameters of the controller can vary according to the operating conditions of the system, this variation is abrupt because they depend on fixed parameter controllers previously designed for some operation points. The fuzzy supervision is inserted in order to attenuate these abrupt changes, weighting the values of the static controllers to find a new set of parameters at each instant of time. Takagi-Sugeno fuzzy systems developed on Matlab ${ }^{\circledR}$ Fuzzy Toolbox were used to update these controllers. Through the analysis of performance indexes, it was concluded that the insertion of fuzzy supervision improves the performance of the systems, reducing the cumulative error when compared to the traditional gain scheduling.
\end{abstract}

Keywords-fuzzy control, PID, non-linear systems, gain scheduling

Resumo-Neste trabalho são comparados o desempenho entre controladores por ganho programado tradicional e uma rede de controladores locais com supervisão fuzzy. As análises são realizadas em dois sistemas: uma válvula não linear e uma planta Motor-Tacogerado. O ganho programado é uma estratégia de controle na qual os parâmetros do controlador podem variar em função das condições de operação do sistema, esta variação é abrupta, pois as condições de operação são divididas em faixas associadas a controladores de parâmetros fixos previamente projetados. A supervisão fuzzy é inserida de forma a atenuar essas mudanças bruscas, ponderando os valores dos controladores estáticos para encontrar um novo conjunto de parâmetros a cada instante de tempo. Sistemas fuzzy de TakagiSugeno desenvolvidos na toolboox de fuzzy do Matlab® foram utilizados para a atualização desses controladores. Através da análise de índices de desempenho, concluiu-se que a inserção da supervisão fuzzy melhora o desempenho dos sistemas, reduzindo o erro acumulado, quando comparados com o ganho programado tradicional

Palavras-chave-Controle Fuzzy, PID, sistemas não lineares, ganho programado

\section{INTRODUÇÃO}

A teoria de controle linear se preocupa principalmente com o estudo de sistemas lineares invariantes no tempo (LTI), cuja forma é

$$
\dot{x}=A x
$$

Em que $x$ é um vetor de estados e $A$ é a matriz que representa o sistema. As propriedades dos sistemas LTI são: se $A$ é não singular, o sistema linear tem um único ponto de equilíbrio; o ponto de equilíbrio é estável se todos os autovalores de $A$ têm parte real negativa, independente das condições iniciais; e na presença de uma entrada externa $u(t)$, ou seja:

$$
\dot{x}=A x+B u
$$

a resposta do sistema tem as seguintes propriedades: satisfaz o princípio da superposição; a estabilidade assintótica do sistema implica num comportamento do tipo BIBO (bounded input, bounded output) na presença de u, i. e., uma entrada estável, induz uma saída estável. E por último, uma entrada senoidal gera uma saída senoidal de mesma frequência [1].

Por essa simplicidade, as metodologias de controle linear também são aplicadas a plantas não lineares em uma região restrita de operação (range), utilizando técnicas de linearização nestes pontos. Porém as limitações das metodologias lineares de controle são o pequeno range de operações e a existência de não linearidades que não podem ser linearizadas devido sua natureza descontínua. Para superar estas dificuldades são utilizadas metodologias de controle não linear que tem se destacado por superar estas características, mantendo sua simplicidade de projeto. Alguns exemplos de metodologias de controle não linear são as técnicas de controle adaptativo ou aquelas que incluem estratégias de inteligência computacional, como redes neurais e lógica fuzzy[1].

Neste artigo serão comparadas as técnicas de ganho programado (GP), desenvolvida durante o século XX e ainda em uso em ambiente industrial [2] e a técnica fuzzy Rede de Controladores Locais (RCL), que combina controladores lineares com uma supervisão Fuzzy no modelo TakagiSugeno, apresentada em [3]. O trabalho está dividido em: Seção 2, na qual são discutidas as técnicas de controle utilizadas; Seção 3, onde são apresentados os sistemas analisados; Em seguida, na Seção 4 são apresentados e discutidos os resultados obtidos durante os testes e, por fim, as conclusões são apresentadas na Seção 5.

\section{GANHO PROGRAMADO E REDE DE CONTROLADORES LOCAIS COM SUPERVISÃO FUZZY}

\section{A. Ganho programado}

A estratégia de controle por ganho programado é aplicada quando o sistema excursiona sobre um intervalo amplo de condições de operação. Para aplicá-la são escolhidos diferentes pontos (faixas) de operação e projetados controladores a parâmetros fixos para cada ponto escolhido. A questão principal do projeto por ganho programado é escolher quais as variáveis do processo servirão de referência para a troca das faixas de operação. Esta escolha é feita a partir do conhecimento do projetista a respeito do processo. 
Neste artigo, os controladores a parâmetros fixos utilizados durante o projeto de ganho programado foram controladores PID obtido por cancelamento dinâmico [4]. Esta estratégia visa o cancelamento da dinâmica original de malha aberta da planta e a obtenção de uma resposta de malha fechada do sistema controlado de primeira ordem, cujo equivalente discreto é:

$$
G_{m f}\left(z^{-1}\right)=\frac{\left(1-z_{d}\right) z^{-1}}{1-z_{d} z^{-1}}
$$

Em que $z_{d}$ é o polo desejado de malha fechada e cujo valor pode ser associado à constante de tempo de malha fechada desejada, $\tau_{m f}$, a partir do modelo SOPDT (Second Order Plus Dead Time):

$$
z_{d}=e^{\frac{T_{s}}{\tau_{m f}}}
$$

sendo $T_{S}$ o período de amostragem. O projeto visa a definição de um controlador PID discreto descrito por:

$$
\frac{U\left(z^{-1}\right)}{E\left(z^{-1}\right)}=\frac{S\left(z^{-1}\right)}{\Delta}=\frac{\left(s_{0}+s_{1} z^{-1}+s_{2} z^{-2}\right)}{1-z^{-1}}
$$

Cujos parâmetros são:

$$
\begin{gathered}
s_{0}=\frac{\left(1-z_{d}\right)}{b_{0}+b_{1}} \\
s_{1}=a_{1} s_{0} \\
s_{2}=a_{2} s_{0}
\end{gathered}
$$

\section{B. Redes de controladores locais com supervisão fuzzy}

Segundo [2], a principal deficiência da estratégia por ganho programado é a queda de desempenho durante a transição entre as faixas de operação. Devido a isso [3] propuseram uma abordagem fuzzy para resolver este problema quando aplicado a sistemas de potência. Os sistemas fuzzy, desenvolvidos a partir dos trabalhos do matemático L. A. Zadeh e extensamente discutidos em [5], são capazes de lidar com informações incertas, ou qualitativas, que podem ser representadas por variáveis linguísticas, de forma análoga a capacidade de inferência humana. Para isso, se baseiam no princípio de pertinência de um elemento num conjunto. Enquanto na lógica clássica existem apenas dois estados possíveis para um elemento, $\in$ ou $\notin$, na lógica fuzzy um elemento tem um grau de pertinência que varia de 0 a $100 \%$.

A partir desse conceito foram desenvolvidos os sistemas fuzzy, compostos por entradas, saídas, a máquina de inferência e as regras, que juntas permitem extrair conhecimento a partir de variáveis linguísticas. As entradas do sistema são valores processados pela máquina de inferência fuzzy, a partir de regras com um formato SE-ENTÃO, resultando nos valores de saída.

A adição do fuzzy ao ganho programado possibilita uma maior flexibilidade do controlador, permitindo que nas zonas de interseção entre as faixas de operação, os parâmetros utilizados sejam uma combinação linear de alguns dos controladores propostos. Isso evita as variações abruptas de controladores, aumentando a eficiência do sistema de controle como um todo.

O projeto de um sistema fuzzy consiste na definição dos conjuntos fuzzy de entrada (e de saída, quando necessário), do conjunto de regras e na escolha da máquina de inferência.
Neste trabalho foi utilizada a máquina do tipo Takagi-Sugeno [5].

\section{PRocessos UtILIZADOS}

Foram escolhidos dois sistemas para servirem de base de comparação. Eles são um sistema não linear descrito em [2] e uma planta real construída e utilizada para fins didáticos no Laboratório de Controle de Sistemas (LACOS) da UFPA.

\section{A. Válvula não linear}

A válvula utilizada nos testes foi retirada de [2] e é descrita pela seguinte função de transferência de tempo contínuo:

$$
G(s)=\frac{v}{s^{3}+3 s^{2}+2 s+1}
$$

Em que $v=u^{4}$ e $u$ é o sinal de entrada da planta. Este processo possui uma não linearidade que torna sua resposta bastante diferente dependendo do valor da entrada. Para exemplificar, a Figura 1 contém a resposta deste sistema para diferentes valores de referência $(0,3 ; 1 ; 5)$. Para a realização desse teste o sistema foi controlado por um controlador PI cujos parâmetros foram $K_{p}=0,15$ e $K_{i}=0,15$.

\section{Sistema Não Linear}

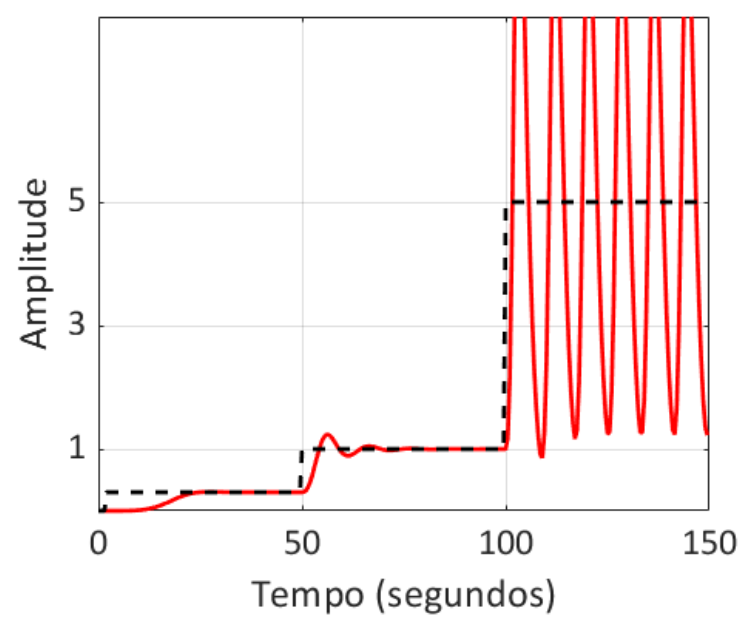

Fig. 1. Resposta da válvula não linear para diferentes referências.

O intervalo de [0:5] foi definido como os limites de variação para a referência, pois foi entendido como adequado para representar a variabilidade de resposta da planta, como pode ser visto na Figura 1. Após alguns testes, foram escolhidos quatro pontos de operação $[0,5 ; 1,5 ; 3 ; 4,5]$ e para cada um deles foi identificado um modelo discreto $\left(T_{s}=\right.$ $0,5 s$ ) de segunda ordem com a técnica dos mínimos quadrados recursivos (MQR), descrito em [6]. A estrutura do modelo de segunda ordem é mostrada na equação 8 e os modelos obtidos para cada ponto são descritos nas equações 9 a 12 .

$$
\begin{gathered}
G\left(z^{-1}\right)=\frac{b_{0}+b_{1} z^{-1}}{1+a_{1} z^{-1}+a_{2} z^{-2}} \\
G_{0,5}\left(z^{-1}\right)=\frac{0,003+0,011 z^{-1}}{1+1,529 z^{-1}+0,644 z^{-2}} \\
G_{1,5}\left(z^{-1}\right)=\frac{0,536+0,261 z^{-1}}{1-1,42 z^{-1}+0.547 z^{-2}}
\end{gathered}
$$




$$
\begin{aligned}
G_{3,0}\left(z^{-1}\right) & =\frac{0,388+1,4341 z^{-1}}{1-1,568 z^{-1}+0,6355 z^{-2}} \\
G_{4,5}\left(z^{-1}\right) & =\frac{1,311+4,84 z^{-1}}{1-1,568 z^{-1}+0,6355 z^{-2}}
\end{aligned}
$$

A partir destes modelos foram projetados os controladores para cada ponto de operação utilizando a técnica do cancelamento dinâmico, resultando nos seguintes controladores:

$$
\begin{aligned}
& C_{0,5}=\frac{0,3955-0,6049 z^{-1}+0,2548 z^{-2}}{1-z^{-1}} \\
& C_{1,5}=\frac{0,2313-0,3359 z^{-1}+0,1265 z^{-2}}{1-z^{-1}} \\
& C_{3,0}=\frac{0,1726-0,2707 z^{-1}+0,1097 z^{-2}}{1-z^{-1}} \\
& C_{4,5}=\frac{0,1457-0,2285 z^{-1}+0,0926 z^{-2}}{1-z^{-1}}
\end{aligned}
$$

Após a definição dos controladores foram definidas as faixas de atuação para cada controlador, que podem ser vistas na Tabela 1.

TABLE I. FAIXAS DE OPERAÇÃo DOS CONTROLADORES - VÁlVULA

\begin{tabular}{|c|c|}
\hline $\boldsymbol{C}_{\mathbf{0 , 5}}$ & A partir de $u=0$ até $u=1,1$ \\
\hline $\boldsymbol{C}_{\mathbf{1 , 5}}$ & A partir de $u>1,1$ até $u=2,25$ \\
\hline $\boldsymbol{C}_{\mathbf{3}, \mathbf{0}}$ & A partir de $u>2,25$ até $u=3,75$ \\
\hline $\boldsymbol{C}_{\mathbf{4}, \mathbf{5}}$ & A partir de $u>3,75$ até $u=5$ \\
\hline
\end{tabular}

Para a construção da RCL foram definidos os conjuntos fuzzy de entrada correspondentes a cada faixa de operação e as regras do sistema fuzzy. Os conjuntos foram denominados como: Baixo (B), Médio (M), Alto (A) e Muito Alto (MA) e podem ser visualizados na Figura 2, enquanto as regras foram as seguintes:

1) Se o sinal de referência pertencer ao conjunto B, ou seja, $u \leq 1,5$, então:

$$
s_{0}=C_{0,5}(1) ; s_{1}=C_{0,5}(2) ; s_{2}=C_{0,5}(3)
$$

2) Se o sinal de referência pertencer ao conjunto $M$, ou seja, $0,5 \leq u \leq 3$, então:

$$
s_{0}=C_{1,5}(1) ; s_{1}=C_{1,5}(2) ; s_{2}=C_{1,5}(3)
$$

3) Se o sinal de referência pertencer ao conjunto A, ou seja, $1,5 \leq u \leq 4,5$, então:

$$
s_{0}=C_{3,0}(1) ; s_{1}=C_{3,0}(2) ; s_{2}=C_{3,0}(3)
$$

4) Se o sinal de referência pertencer ao conjunto $A$, ou seja, $3 \leq u \leq 5$, então:

$$
s_{0}=C_{4,5}(1) ; s_{1}=C_{4,5}(2) ; s_{2}=C_{4,5}(3)
$$

A Figura 2 contém as funções de pertinência correspondentes as faixas de valores de entrada da válvula. Dependendo do valor de referência, as regras são ativadas e os valores dos parâmetros de saída são calculados a partir daqueles definidos nas equações 13 a 16.

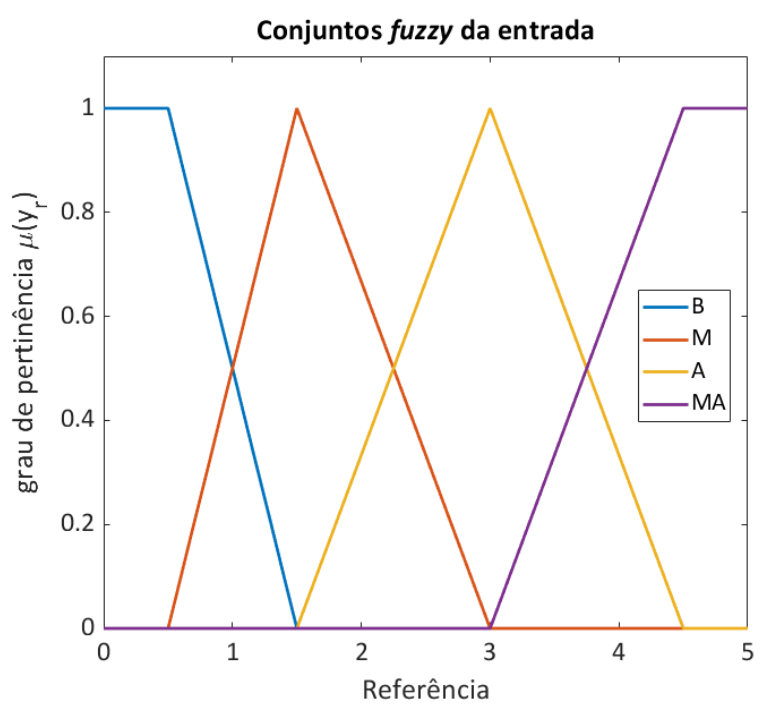

Fig. 2. Funções de pertinência para válvula não linear.

\section{B. Motor Tacogerador}

Para avaliar o desempenho do controlador proposto em um sistema real, foi escolhido a planta Motor Tacogerador (MTG), localizada no Laboratórios de Controle de Sistemas (LACOS) na UFPA (Figura 3). O MTG consiste em duas máquinas de corrente contínua conectadas pelos eixos. A entrada do sistema é a tensão de atuação em um dos motores, enquanto a saída é a tensão gerada pela segunda máquina, funcionando com gerador. Apesar da simplicidade, devido as características construtivas do sistema, ele apresenta comportamento não linear, tendo um ganho menor em baixas tensões e maior em tensões mais altas, e por isso foi escolhido para os testes. A interface entre o sistema e o computador é feita por um Arduino Uno, o que restringe a tensão de leitura e atuação a um intervalo de 0 a $5 \mathrm{~V}$.

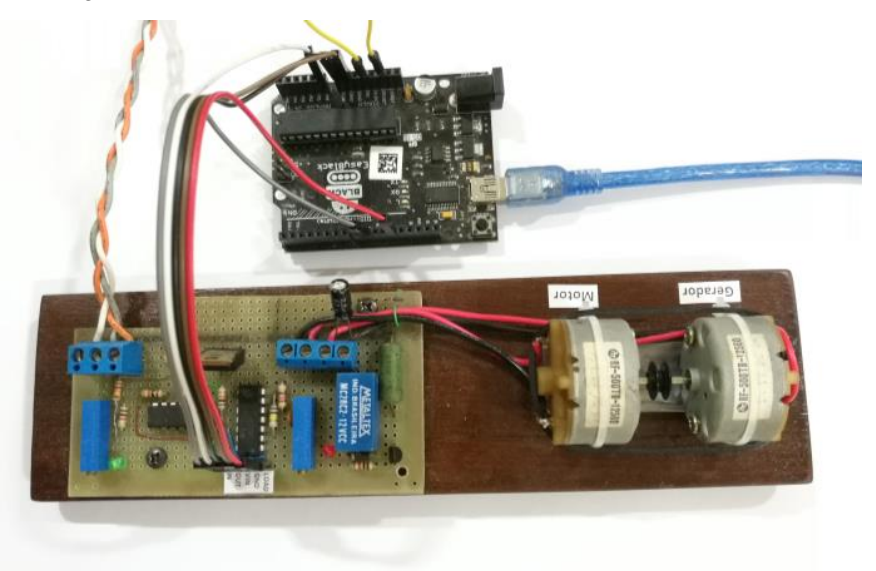

Fig. 3. Motor Tacogerador utilizado nos testes.

Para este processo foram escolhidos três pontos de operação: 1; 2,5 e 4. E em seguida foi realizada a identificação dos modelos em cada ponto de operação, com um tempo de amostragem $T_{s}=0,05 \mathrm{~s}$. Novamente foram escolhidos modelos de segunda ordem com a estrutura descrita na equação (8) e os resultados encontrados são apresentados nas equações 17 a 19.

$$
G_{1,0}\left(z^{-1}\right)=\frac{0,039+0,184 z^{-1}}{1+0,804 z^{-1}+0,094 z^{-2}}
$$




$$
\begin{aligned}
G_{2,5}\left(z^{-1}\right) & =\frac{0,536+0,261 z^{-1}}{1-0,7713 z^{-1}-0.033 z^{-2}} \\
G_{4,0}\left(z^{-1}\right) & =\frac{-0,008+0,376 z^{-1}}{1-0,574 z^{-1}-0,0862 z^{-2}}
\end{aligned}
$$

A partir dos modelos identificados foram projetados os controladores para cada ponto de operação. A técnica de projeto utilizada foi novamente a do cancelamento dinâmico, cuja estrutura está descrita nas equações 3 a 6 . Os controladores encontrados para cada ponto de operação são apresentados nas equações 20 a 22 .

$$
\begin{aligned}
& G_{1,0}\left(z^{-1}\right)=\frac{1,392-1,12 z^{-1}-0,131 z^{-2}}{1-z^{-1}} \\
& G_{2,5}\left(z^{-1}\right)=\frac{0,43-0,332 z^{-1}-0,014 z^{-2}}{1-z^{-1}} \\
& G_{4,0}\left(z^{-1}\right)=\frac{0,361+0,207 z^{-1}+0,03 z^{-2}}{1-z^{-1}}
\end{aligned}
$$

As faixas de atuação para os controladores do ganho programado do MTG estão definidas na Tabela 2.

TABLE II. FaIXAS De OPERAÇão Dos CONTROLADORES - MTG

\begin{tabular}{|l|c|}
\hline $\boldsymbol{C}_{\mathbf{1 , 0}}$ & A partir de $u=0$ até $u=1,75$ \\
\hline $\boldsymbol{C}_{\mathbf{2 , 5}}$ & A partir de $u>1,75$ até $u=3,25$ \\
\hline $\boldsymbol{C}_{\mathbf{4 , 0}}$ & A partir de $u>3,25$ até $u=5$ \\
\hline
\end{tabular}

Para a construção da RCL foram definidos os conjuntos fuzzy de entrada correspondentes a cada faixa de operação e também as regras do sistema fuzzy. Na figura 4 são vistas as três funções de pertinência correspondentes as faixas de valores da tensão de entrada do sistema. Estas faixas cobrem a extensão de valores possíveis para a referência e foram definidas como: Baixo (B), Médio (M), Alto (A).

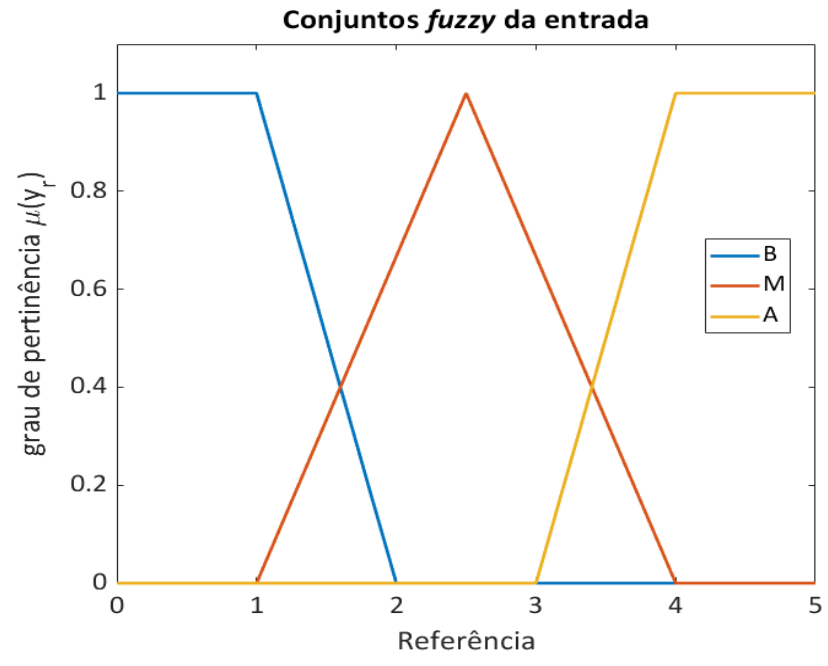

Fig. 4. Funções de pertinência para o sistema MTG.

$\mathrm{E}$ as regras foram definidas como:

1) Se o sinal de referência pertencer ao conjunto B, ou seja, $u \leq 2$, então:

$$
s_{0}=C_{1,0}(1) ; s_{1}=C_{1.0}(2) ; s_{2}=C_{1,0}(3)
$$

2) Se o sinal de referência pertencer ao conjunto $M$, ou seja, $1 \leq u \leq 4$, então:

$$
s_{0}=C_{2,5}(1) ; s_{1}=C_{2,5}(2) ; s_{2}=C_{2,5}
$$

3) Se o sinal de referência pertencer ao conjunto A, ou seja, $3 \leq u \leq 5$, então:

$$
s_{0}=C_{4,0}(1) ; s_{1}=C_{4,0}(2) ; s_{2}=C_{4,0}(3)
$$

\section{Resultados E Discussões}

Para analisar o desempenho do controlador proposto, as plantas foram submetidas a três testes de rastreamento de referência:: o primeiro deles consistiu de uma série de variações do tipo degrau; os outros dois testes foram referências do tipo rampa - crescente e decrescente. A análise foi feita a partir de índices de desempenho como de praxe na área de controle. Os índices escolhidos foram a variância $\sigma^{2}$ do sinal de controle, a integral do erro quadrático (ISE) em sua versão discreta, i.e. o somatório do quadrado do erro em cada amostra

$$
I S E=\sum_{1}^{n i t} e(k)^{2}
$$

o TVC (do inglês, Total Variation Control) que ilustra as mudanças no sinal de controle e consequentemente o esforço desempenhado pelo regulador

$$
T V C=\sum_{1}^{n i t} \Delta u(k)
$$

em que nit é o número de amostras durante a simulação.

\section{A. Válvula não linear}

O primeiro teste a que a válvula foi submetida foi $o$ seguimento de referências do tipo degrau. Os pontos de referência foram escolhidos para explorar as deficiências do Ganho Programado, sendo localizados próximos aos pontos de mudança de faixa de operação. Isto foi feito para analisar a diferença de desempenho entre as técnicas nestas regiões. Os valores escolhidos para os degraus foram: $[\mathbf{3 , 2 5} ; \mathbf{1 , 0 5} ; \mathbf{3 , 8}$; 2,25].

Os resultados da simulação podem ser vistos na Figura 5. A parte superior contém as respostas do sistema quando controlados pelo Ganho Programado (vermelho) e pelo RCL fuzzy (azul), enquanto a parte inferior contém o sinal de controle aplicado à planta.

$\mathrm{Na}$ Figura 5 percebe-se o desempenho melhor da técnica fuzzy em relação ao GP na resposta do sistema. Porém a amplitude do sinal de controle utilizado pelo RCL foi maior. Os índices de desempenho foram reunidos na Tabela $3 \mathrm{e}$ ilustram o ganho do RCL fuzzy em relação ao erro da resposta e a também a variação do controle. A variância do sinal de controle do sistema fuzzy foi melhor que a do ganho programado regular, sendo vinte quatro vezes menor que no GP, isso implica em um menor desgaste num sistema real, cujos ruídos podem influenciar numa resposta mais oscilatória do sistema, reduzindo a durabilidade da planta como um todo e acarretando em prejuízos financeiros. 

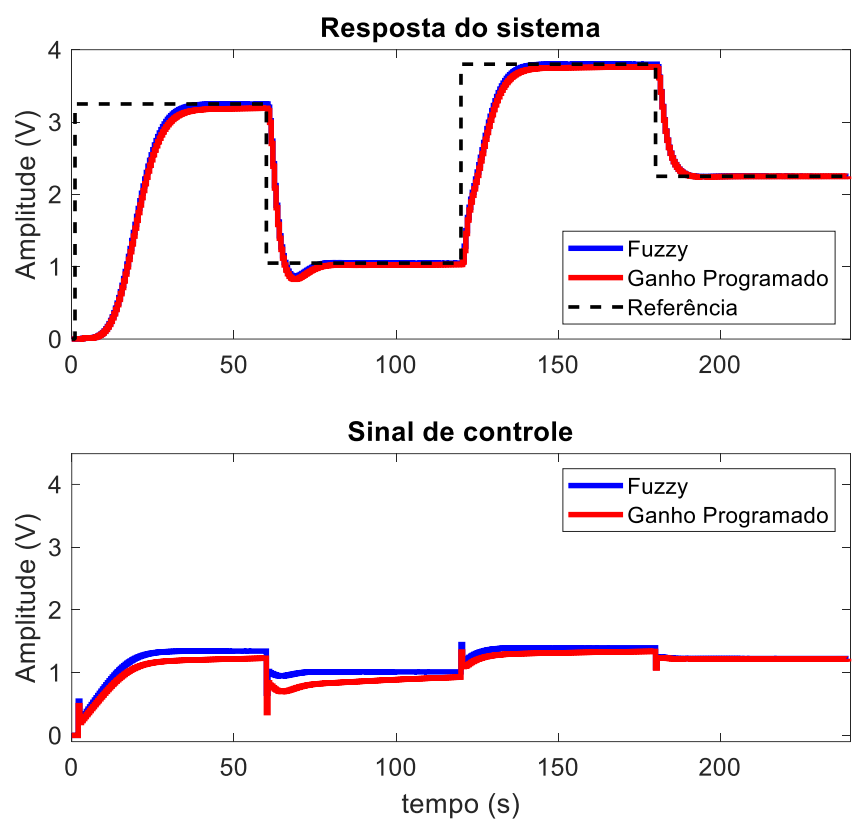

Fig. 5. Resposta da válvula não linear a mudanças de referência do tipo degrau.

O segundo teste foi a exposição da planta a uma entrada do tipo rampa crescente, iniciando em 1 e parando em 4 . A Figura 6 contém a resposta do sistema durante o teste, assim como o esforço de controle utilizado. A partir da análise gráfica não fica clara qualquer superioridade do RCL em relação a resposta do sistema controlado pelo GP, inclusive a análise do índice ISE na Tabela 3 indica que o GP foi superior, porém novamente a variância do sinal de controle do sistema fuzzy foi mais de dezenove vezes menor.
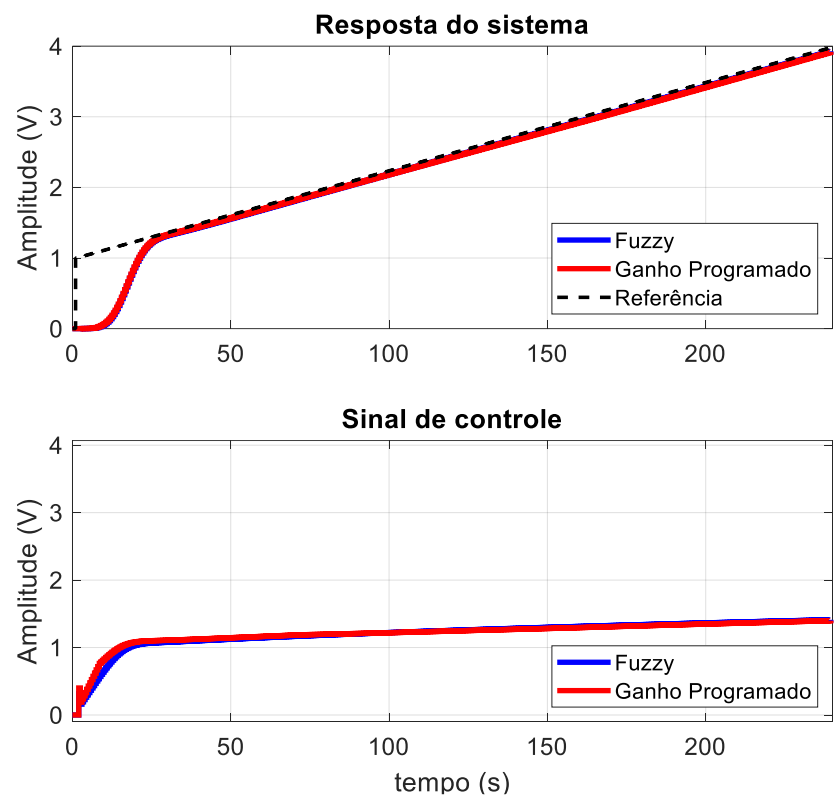

Fig. 6. Resposta da válvula não linear uma entrada rampa crescente.

O último teste com a válvula foi a resposta a entrada do tipo rampa decrescente e os resultados são vistos na Figura 7. Nesse teste o RCL fuzzy teve um menor erro acumulado e menor variância no sinal de controle, enquanto o índice TVC foi menor para o ganho programado.
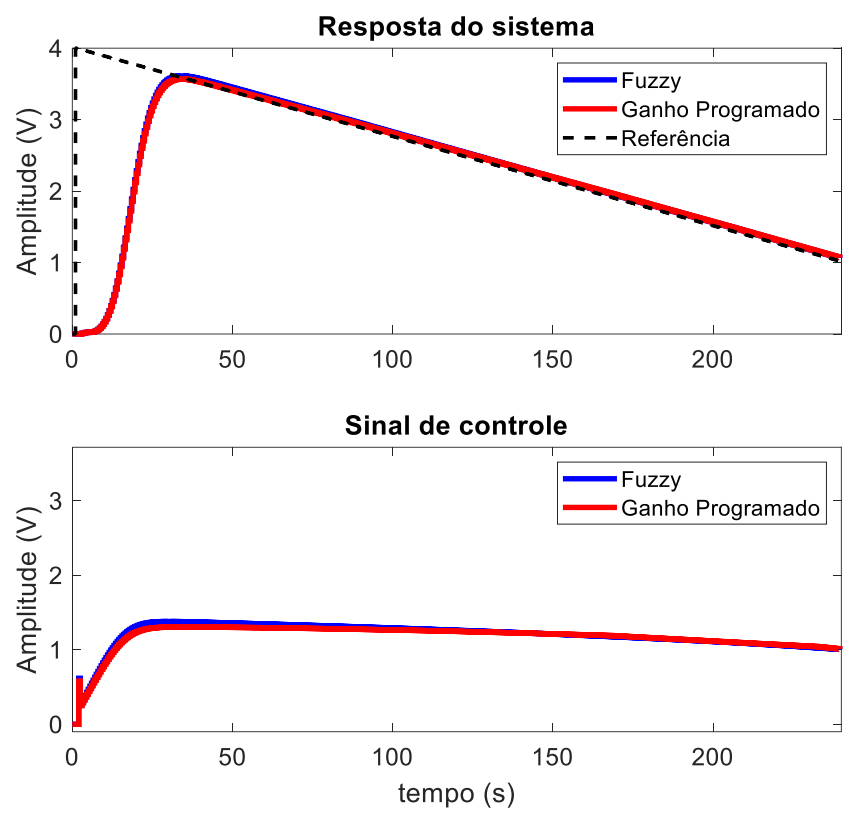

Fig. 7. Resposta da válvula não linear a uma entrada do tipo rampa decrescente.

TABLE III. ÍNDICES DE DESEMPENHO TESTES VÁLVULA

\begin{tabular}{|c|c|c|c|c|}
\hline & ISE & TVC & Variância & $\begin{array}{l}\text { Sinal de } \\
\text { entrada }\end{array}$ \\
\hline Fuzzy & 406,322 & 4,518 & 0,053 & \multirow{2}{*}{$\begin{array}{l}\text { Degraus } \\
\text { variados }\end{array}$} \\
\hline GP & 414,082 & 4,807 & 1,308 & \\
\hline Fuzzy & 32,366 & 1,702 & 0,051 & \multirow{2}{*}{$\begin{array}{c}\text { Rampa } \\
\text { crescente }\end{array}$} \\
\hline GP & 31,829 & 1,818 & 0,971 & \\
\hline Fuzzy & 423,363 & 2,457 & 0,039 & \multirow{2}{*}{$\begin{array}{c}\text { Rampa } \\
\text { decrescent }\end{array}$} \\
\hline GP & 426,392 & 2,264 & 0,0801 & \\
\hline
\end{tabular}

\section{B. Motor tacogerador}

Devido ser um sistema real, o MTG possui ruídos estocásticos, por isso foram realizadas baterias de testes e foram calculados valores médios dos índices de desempenho para o preenchimento da Tabela 4, que reúne os resultados obtidos. Foram aplicados os mesmos três testes anteriores à planta real. Os valores escolhidos para os degraus do primeiro teste foram: $[\mathbf{1} ; \mathbf{3 , 5} ; \mathbf{2} ; \mathbf{4 . 5}$ ], o teste foi realizado 50 vezes para cada técnica e a Figura 8 contém a resposta obtida em um dos testes.

Na Figura 8 é possível enxergar os efeitos dos ruídos na resposta do sistema, trepidações e variações eletromagnéticas são os principais ruídos desta planta. No gráfico percebe-se que o sinal de controle do sistema fuzzy neste teste tem comportamento menos oscilatórias em algumas regiões. Mas apenas a análise dos índices da Tabela 4 permite uma interpretação mais clara das diferenças de desempenho entre as duas estratégias

Para o segundo teste, foi utilizada como entrada uma rampa crescente variando de 1 até 4 . Na Figura 9 é expresso o histórico de variação dos índices de desempenho durante os testes realizados. São expressos os resultados de 50 rodadas de testes com cada uma das técnicas. Este formato expressa melhor a diferença de desempenho do algoritmo RCL fuzzy em relação ao GP do que apenas um recorte da saída do sistema numa única rodada de testes. 

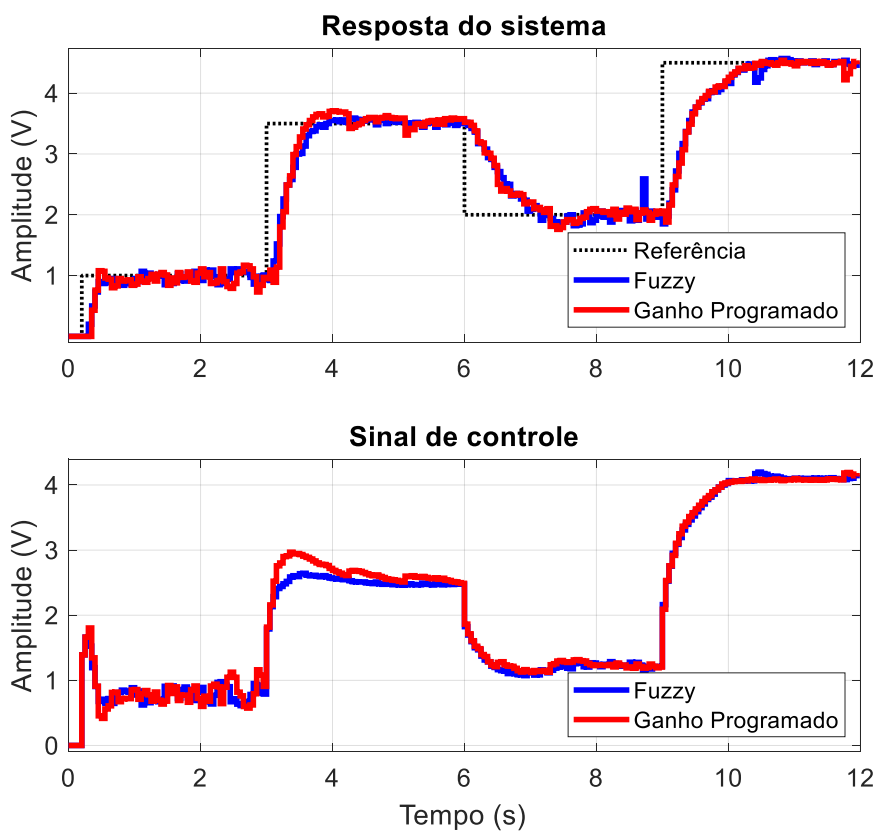

Fig. 8. Resposta do MTG a série de degraus.

TABLE IV. ÍNDICES De DESEMPENHo Testes MTG

\begin{tabular}{|c|c|c|c|c|}
\hline & ISE & TVC & Variância & $\begin{array}{l}\text { Sinal de } \\
\text { entrada }\end{array}$ \\
\hline Fuzzy & 86,683 & 16,106 & 1,528 & \multirow{2}{*}{$\begin{array}{l}\text { Degraus } \\
\text { variados }\end{array}$} \\
\hline GP & 89,683 & 17,744 & 1,561 & \\
\hline Fuzzy & 0,271 & 23,082 & 1,094 & \multirow{2}{*}{$\begin{array}{c}\text { Rampa } \\
\text { crescente }\end{array}$} \\
\hline GP & 0,304 & 26,869 & 1,203 & \\
\hline Fuzzy & 3,514 & 23,839 & 1,183 & \multirow{2}{*}{$\begin{array}{c}\text { Rampa } \\
\text { decrescente }\end{array}$} \\
\hline GP & 3.587 & 26,931 & 1,274 & \\
\hline
\end{tabular}
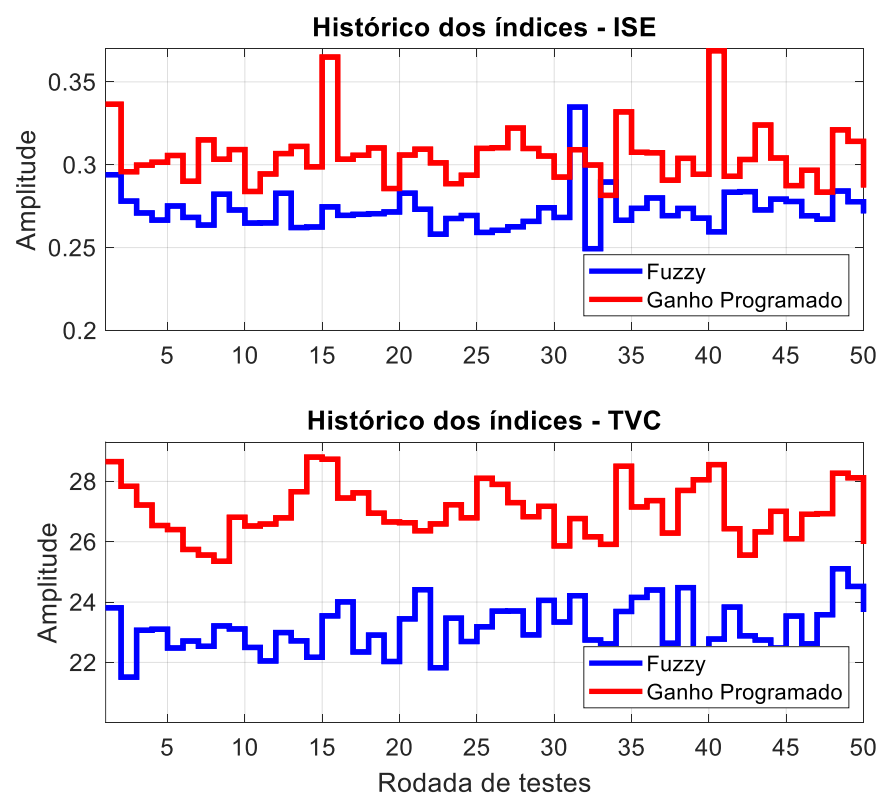

Através da análise dos dados da Figura 9 percebe-se que o sistema fuzzy foi superior em mais de $95 \%$ dos testes no quesito ISE; enquanto no TVC, em todos os testes o GP apresentou mais esforço de controle do que o RCL fuzzy. Isso reflete a eficiência da técnica analisada quando lidando com sistemas ruidosos, assim como a dificuldade que um sistema de controle por ganho programado tem de acompanhar uma referência do tipo rampa. Por fim, a Figura 10 contém o histórico de índices de desempenho para uma entrada do tipo rampa decrescente.

Assim como anteriormente, na Figura 10 percebe-se que o RCL tem melhor desempenho que o GP. Em 75\% dos testes, o ISE do sistema fuzzy foi mais baixo que o da outra metodologia. Enquanto em apenas um dos testes, o TVC do ganho programado obteve um desempenho melhor. Ao analisar os valores da Tabela 4 percebe-se a vitória do RCL em todos os índices da planta real, independente do teste. O índice TVC do RCL foi pelo menos $10 \%$ menor que o do ganho programado, enquanto no ISE e na variância houveram vitórias por uma margem pequena.
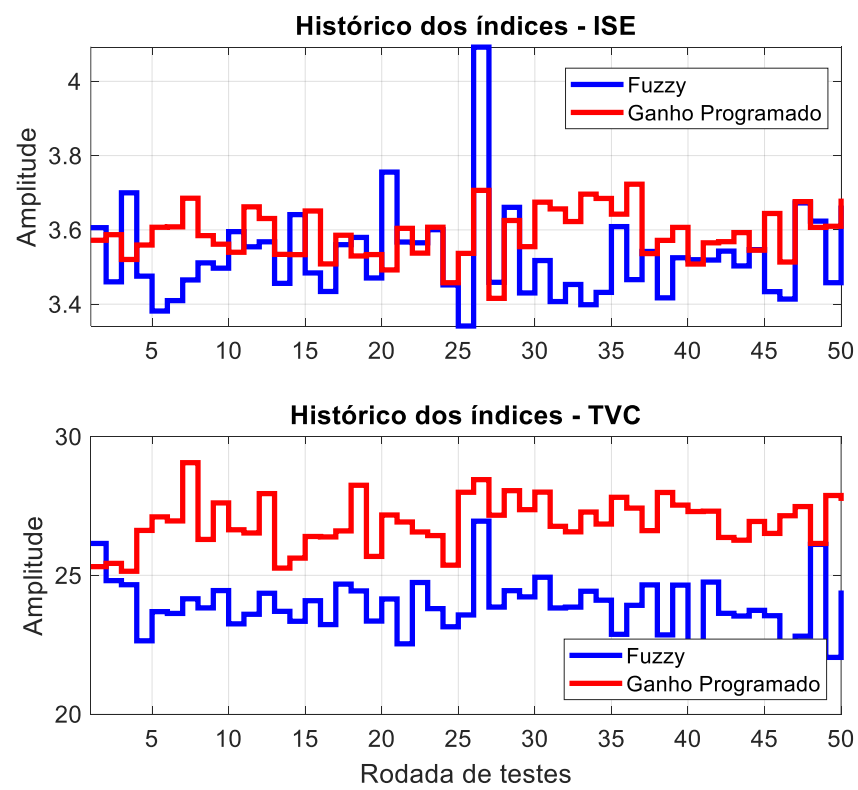

Fig. 10. Variação dos índices de desempenho durante os testes com a entrada do tipo rampa decrescente

\section{CONCLUSÃO}

O presente estudo avaliou o desempenho da técnica rede de controladores locais com supervisão fuzzy em relação a técnica de ganho programado tradicional. Os resultados mostram que a técnica utilizada demonstrou melhor desempenho que o algoritmo de ganho programado nos testes propostos. A inserção das técnicas fuzzy permite que conhecimento a respeito dos sistemas torne o processo de projeto de controladores para plantas não lineares com faixas amplas de operação mais simples, reduzindo o número de pontos de operação e projetos de controladores necessários para o correto funcionamento da planta. A equipe de pesquisa agora busca aplicar as técnicas às demais plantas do laboratório para poder consolidar os resultados desse estudo.

Fig. 9. Variação dos índices de desempenho durante os testes com a entrada do tipo rampa crescente. 


\section{AGRADECIMENTOS}

Os autores agradecem ao suporte do Programa de PósGraduação em Engenharia Elétrica (PPGEE) da Universidade Federal do Pará (UFPA), ao CNPQ e a CAPES.

\section{REFERÊNCIAS}

[1] Slotine. J.J. E.; Li W. (1991) Applied Nonlinear Control. Englewood Cliffs, NJ, USA: Prentice-Hall, Inc.

[2] Aström, K. J.; Wittenmark, B. (2008). Adaptive Control. New York, NY, USA: Prentice-Hall Inc.
[3] Barra Jr, W.; Barreiros, J. A. L.; Costa Jr, C. T.; Ferreira, A. M. D. (2005) Controle Fuzzy Aplicado à Melhoria da Estabilidade Dinâmica em Sistemas Elétricos de Potência, SBA. Sociedade Brasileira de Automática, Brasil, vol. 16, no 2, pp. 173-186.

[4] Araújo, M. S.; Dutra, B. G.; Pinheiro, T. C. F.; Cunha, L. B. (2017). Projeto de Controladores PID e IPD: uma Análise de Desempenho. XIII Simpósio Brasileiro de Automação Inteligente - SBAI, Porto Alegre, RS.

[5] Wang. L. A (1997) Course in Fuzzy Systems and Control. Upper Saddle River, NJ, USA: Prentice-Hall, Inc.

[6] Aguirre, L. A. (2015). Introdução à Identificação de Sistemas: Técnicas Lineares e Não Lineares Aplicadas a Sistemas Reais. $4^{\mathrm{a}}$ ed. Belo Horizonte: Editora UFMG 